\title{
Atmospheric Entry Studies for Uranus
}

Parul Agrawal*!, Gary Allen Jr.!, Helen Hwang, Dinesh Prabhu!, Jose Aliaga, Mark Marley, Kathy McGuire, Loc Huynh, Joseph Garcia (NASA Ames Research Center, Moffett Field, California, U.S.A.) ! ERC

Robert Moses \& Rick Winski

(NASA Langley Research Center, Hampton, Virginia, U.S.A.)

*POC: parul.agrawal-1@nasa.gov

Evgeniy Sklyanskiy
(Jet Propulsion Lab, California, U.S.A)

Phone: (650)-604-3764

\section{Objectives}

- Establish a range of probe atmospheric entry environments based on the Uranus Flagship mission outlined in the Planetary Science Decadal Survey for two launch windows: Year 2021 and 2034.

- Define Uranus entry trade space by performing parametric studies, by varying vehicle mass and size and entry Flight Path Angle (FPA).

- Investigate various trajectory options, including direct ballistic entry and aero-capture entry.

- Identify entry technologies that could be leveraged to enable a viable mission to Uranus that meets science objectives.

\section{Orbital Mechanics}

Target Entry Interface Radius: 28,474 km

Flight Path Angle (FPA): $-19.50^{\circ}$

Entry velocity $\left(\mathrm{V}_{\mathrm{E}}\right)$ for 2043: $21.96 \mathrm{~km} / \mathrm{s}$

$V_{E}$ for 2029: $22.53 \mathrm{~km} / \mathrm{s}$
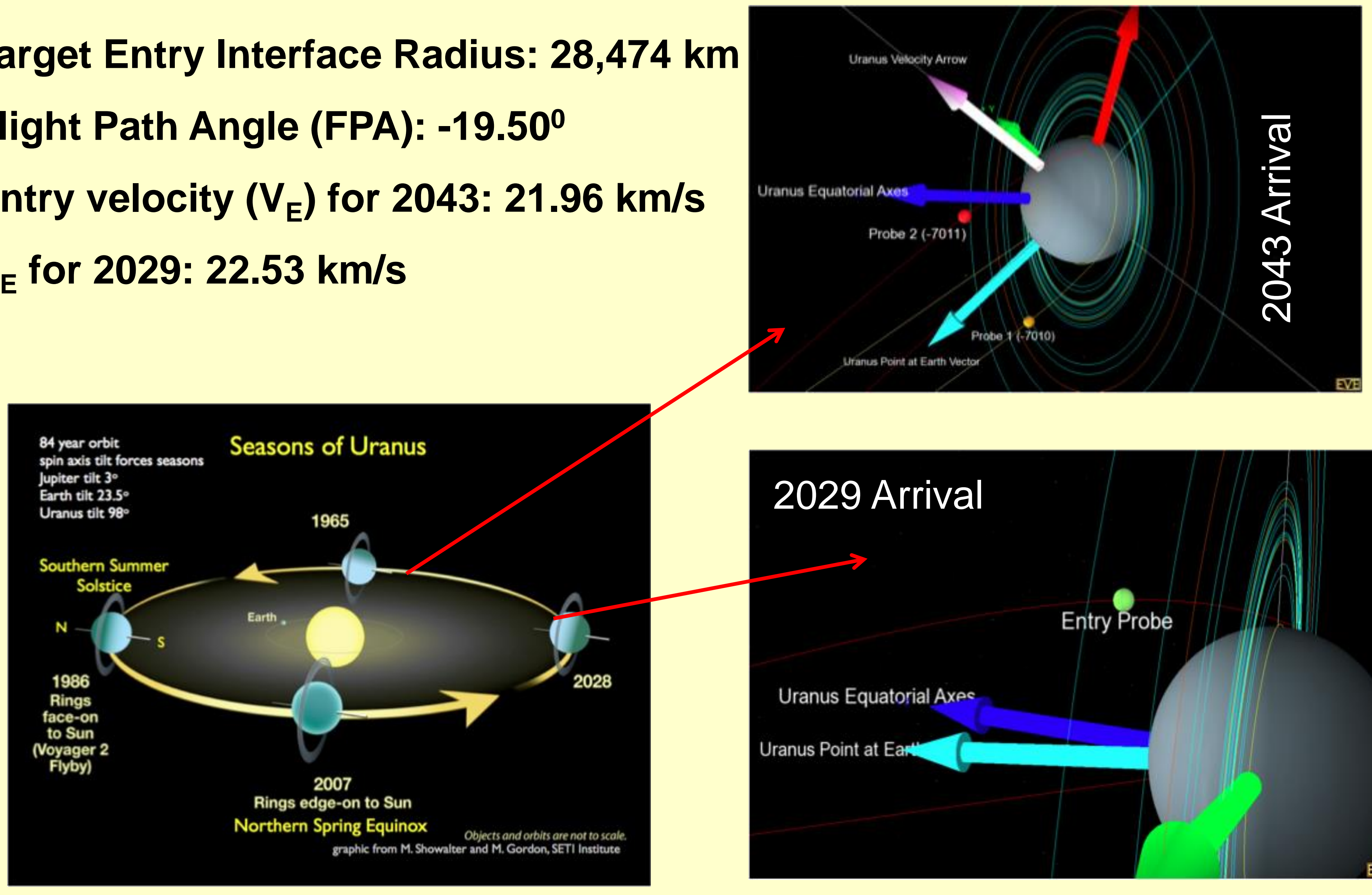

Uranus Orbit and Interplanetary Trajectory

- Earth-Jupiter-Uranus chemical propulsion trajectory was used.

- Various trajectory options were investigated for ring avoidance.

Two arrival windows were selected for detailed entry trade analysis.

\section{Direct Ballistic Entry}

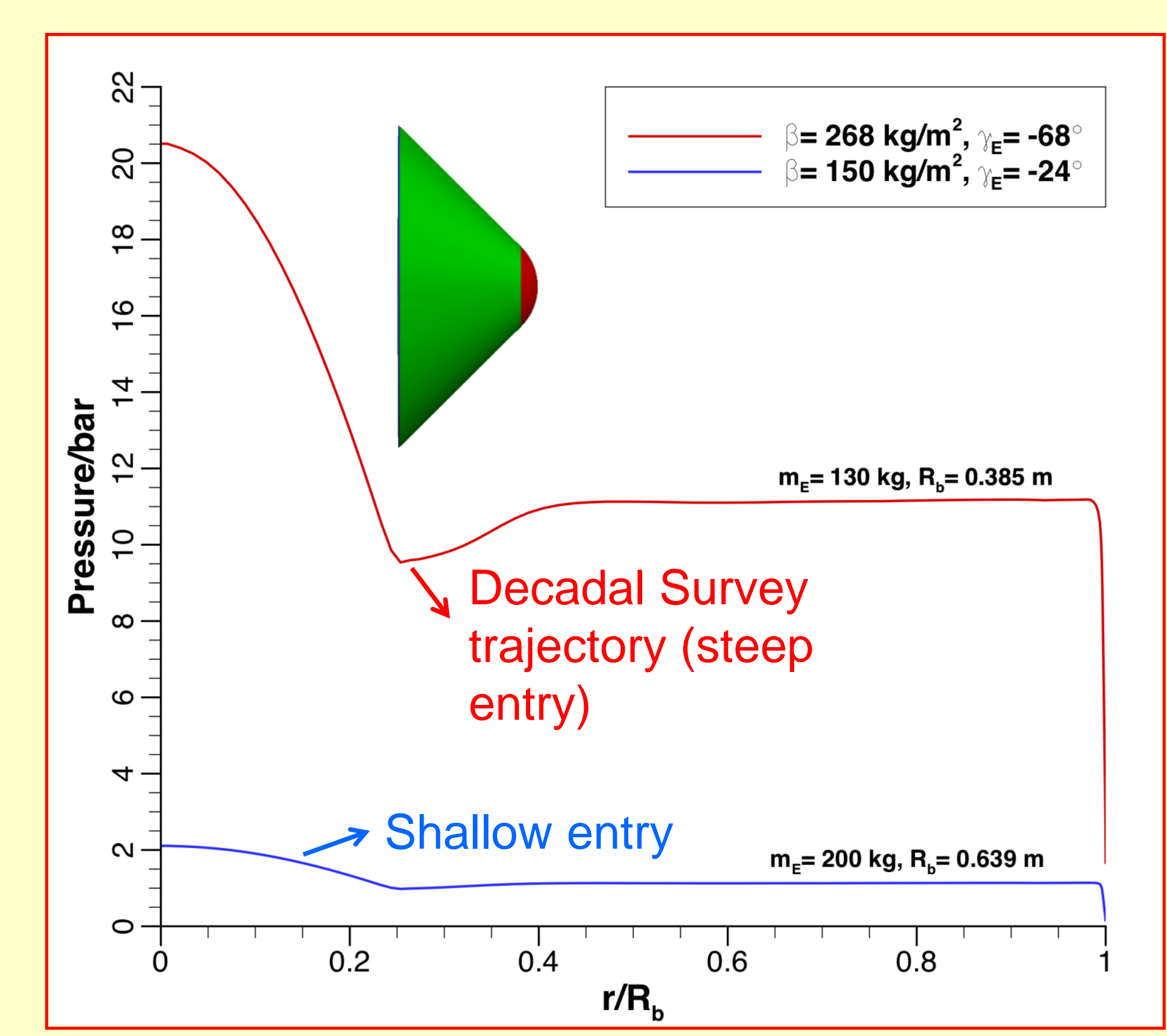

Pressure Distribution

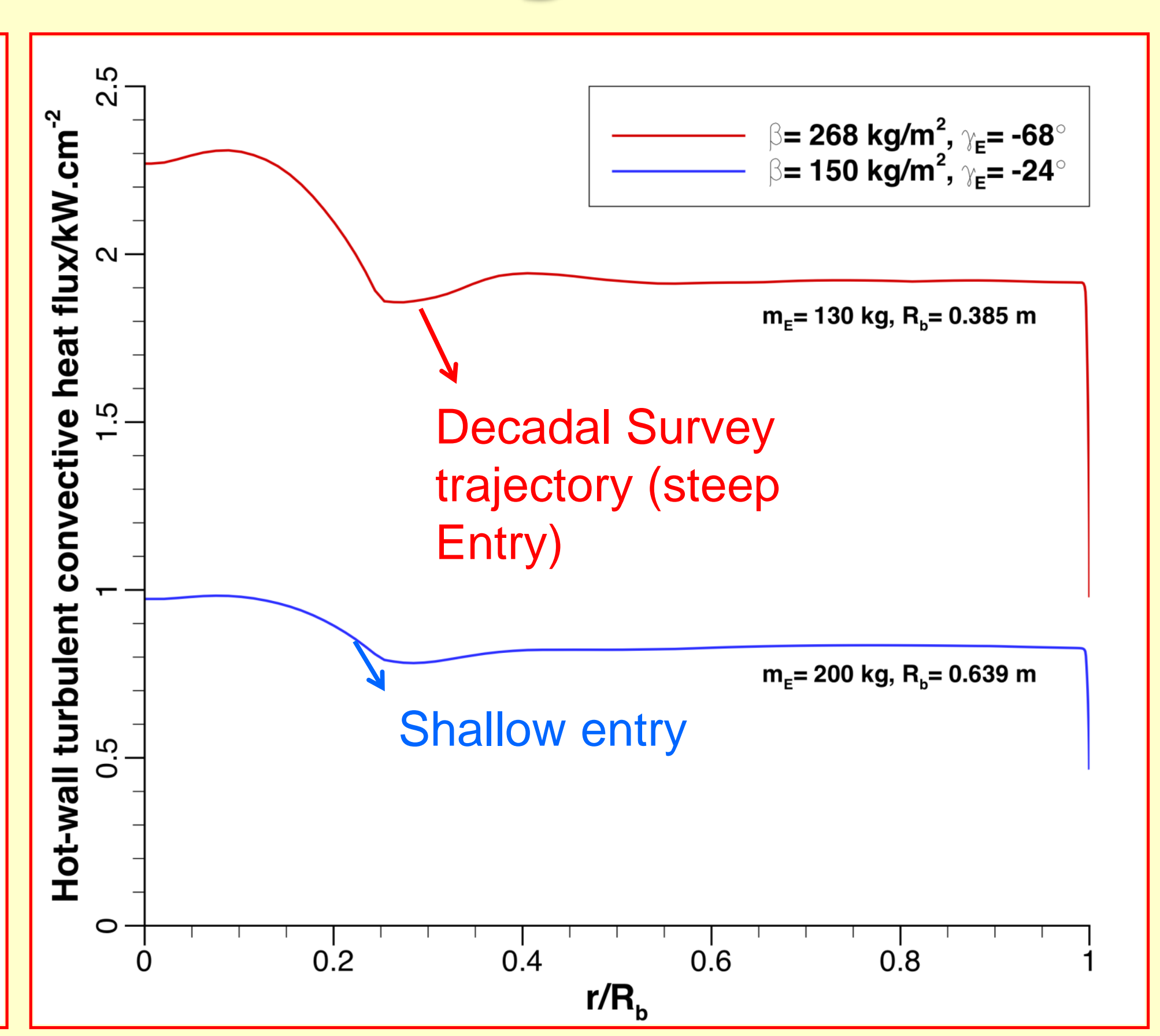

Heat flux Distribution
- For both 2029 and 2043 arrival a range of entry trajectories (several thousands) are generated by varying entry FPA $\left(\gamma_{e}\right)$ for various ballistic coefficients.

- The entry trade space are generated with these trajectories.

- Suitable ranges for flight path angle for various ballistic coefficients $\left(100 \mathrm{~kg} / \mathrm{m}^{2}-300 \mathrm{~kg} / \mathrm{m}^{2}\right)$ are identified, based on G-load, skip-out and pressure constraints.

- Heat flux, heat load, pressure and shear load are estimated and TPS selection and mass calculations are performed.

\section{Atmosphere}

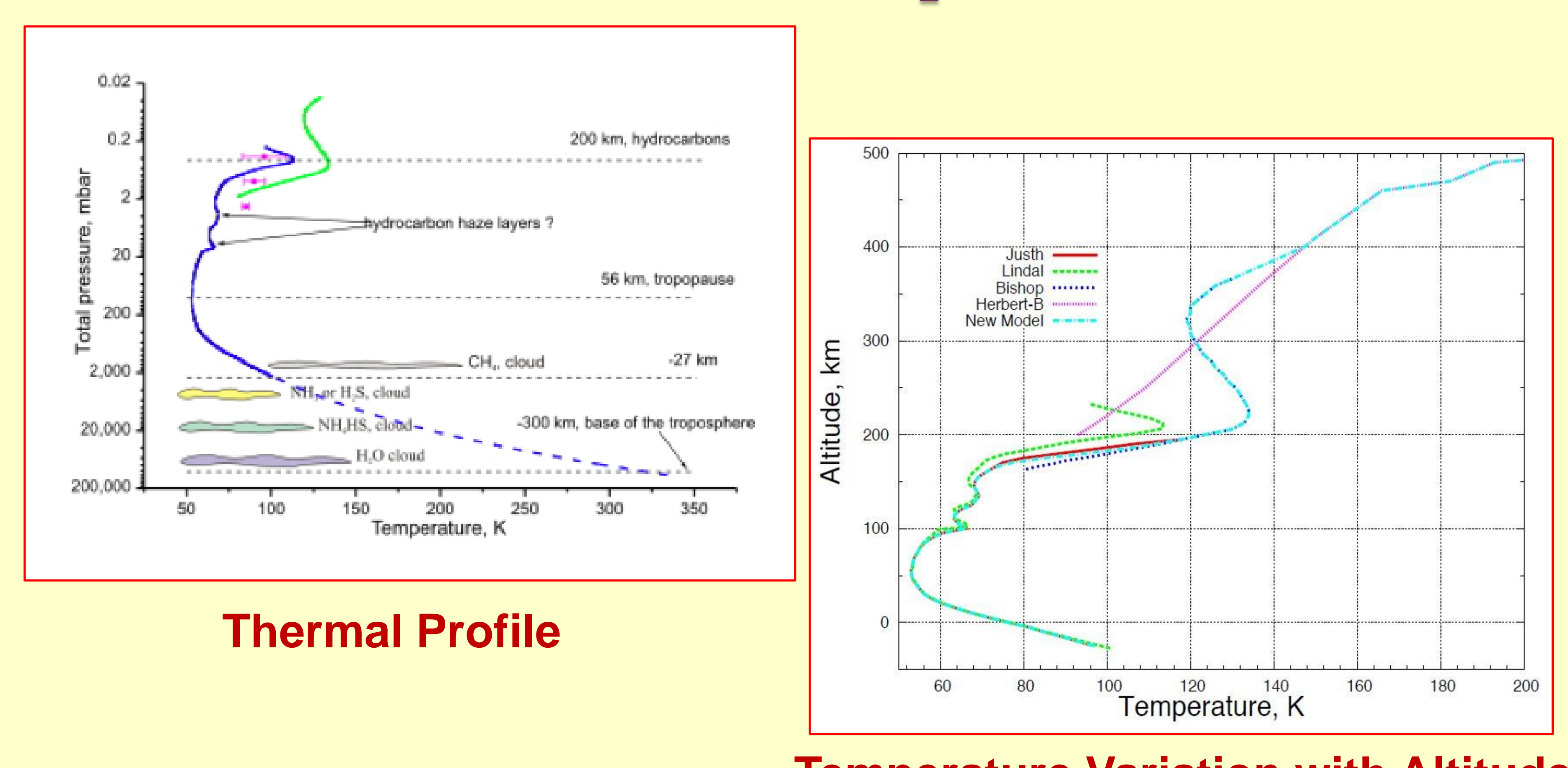

Temperature Variation with Altitude

- The different published atmospheric models are stitched together to construct a "new engineering atmosphere model".

- The ambient temperature at upper altitude is very high (800K); the phenomenon needs investigation.

- The upper atmosphere of Uranus is remarkably dense that influences the entry parameter.

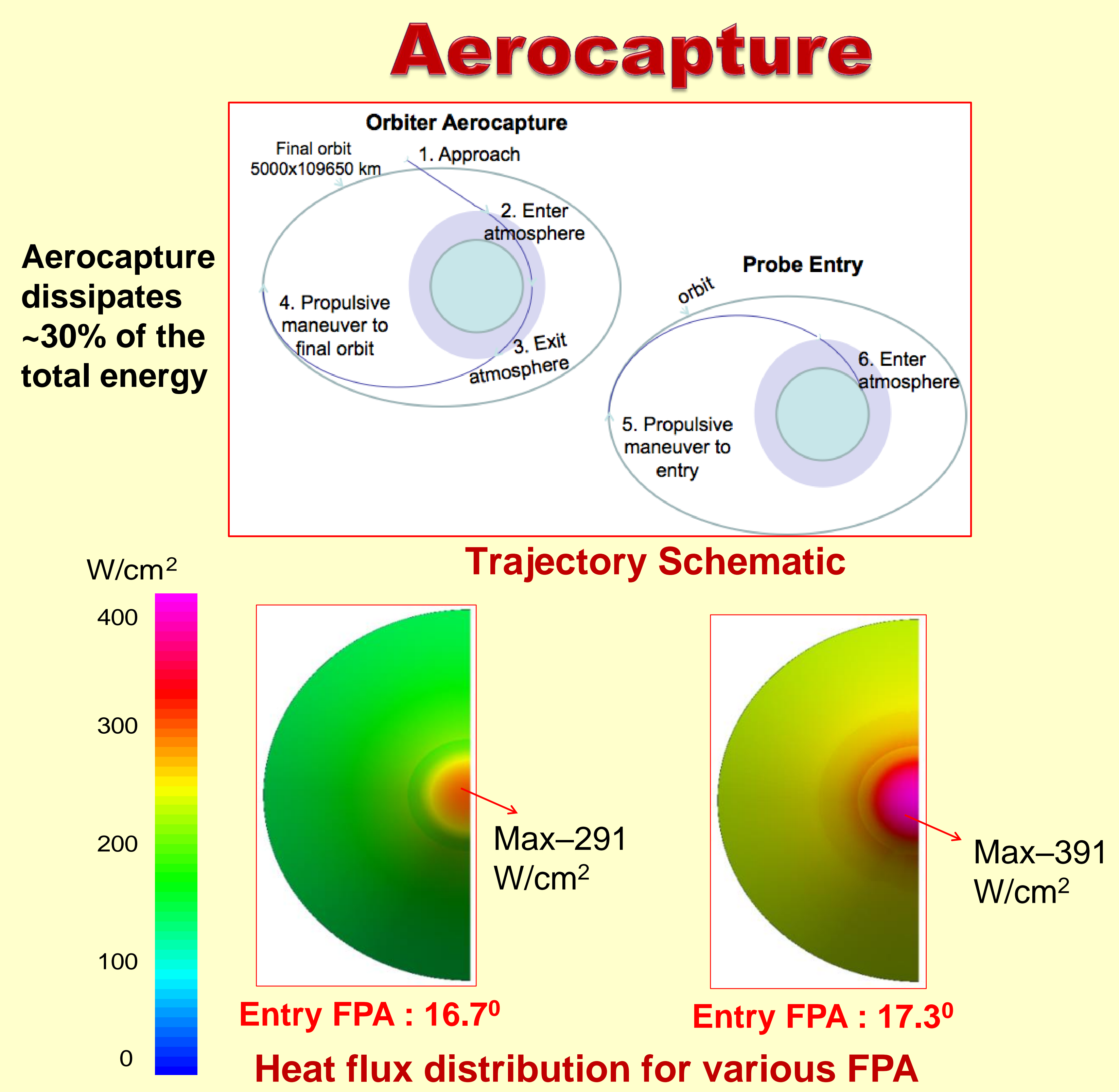

- Entry from orbit would facilitate the use of low density TPS like phenolic impregnated carbon ablator.

- Aerocapture has the potential to save mass as compared to Solar Electric Propulsion (SEP) orbit capture.

Note: Results from this study will be documented in a NASA-TM as well as published in an archival journal in year 2014 